\title{
COMPARATIVE STUDY ON VARIOUS METHODS OF DOA ESTIMATION OF SOURCE TARGET FOR UNDERWATER APPLICATION
}

\author{
Prashil M. Junghare ${ }^{1}$, Nagaraj ${ }^{2}$, Cyril Prasanna Raj $\mathbf{P}^{\mathbf{3}}$, Chandrakala $\mathrm{S}^{\mathbf{4}}$ \\ ${ }^{I}$ Research Scholar, PRIST University, Tamilnadu, India \\ ${ }^{2}$ Student, Department of ECE, The Oxford College of Engineering, Karnataka, India \\ ${ }^{3}$ Professor \& Dean, R\&D, M.S. Engineering College, Karnataka, India \\ ${ }^{4}$ Assistant professor, Department of ECE, The Oxford College of Engineering, Karnataka, India
}

\begin{abstract}
DOA computation is a major issues in several applications. This kinds of application found in remote wireless communication system, radar system, radio astronomy system, sonar system and navigation system. Determination of a DOA computation will be improved using a antenna arrangement by an advanced signal processing procedure techniques. These DOA techniques have improved array antenna arrangements to process various receiving signals at array elements. These methods possess good capability to find a DOA of various sources. This work can elaborate implementation of DOA computation algorithmsMUSIC \&ESPRIT based on a ULA in existence of various environmental noises. Execution of DOA mainly depends on various input parameters like snapshots, No.of array sensors, SNR ratio. Simulation results found shows MUSIC \& ESPRIT performance can be increased efficiently as no. of snapshot, no. of array components, SNR\& angle between 2 target sources are enhanced.
\end{abstract}

Keywords: Uniform Linear Array; DOA (Direction of Arrival); Array Signal Processing; Antenna system; Array elements; Estimation of Signal Parameters via Rotational Invariance Techniques (ESPRIT); Multiple Signal Classification (MUSIC)

\section{INTRODUCTION}

In following current decade's correct perception of DOA of signal has discovered numerous application such as RADAR system \& communication systems, military\& various other applications. For an instance, in case of commercial application it is very essential to find directions of crisis phone call to send an emergency rescue team sent to the exact location of a crisis. In defense application it is always necessary to find direction of threats to help them. If we consider case of a communication systems, goal of DOA estimation is to utilize information obtained at array sensor exhibit with sight direction of coming signals starting with those specified versatile clients. Also consequently the directions from claiming obstruction signals. The results of incoming DOA calculation measures used to vary the weights of an adaptive beam former, provided that a power radiated can be enhanced towards a desired user \& radiation nulls sets in a directions of a arriving incoming signal waves.

If various sources operated in mean time, each source forms a various multipath elements at a receiver end\& thus receiver arrays will find the angle of incoming arriving signal. Other various procedures to computerange of incidents signals on antenna array system \& incidence angle are as follows Capon, Min-norm, Bartlett MUSIC, and
ESPRIT. MUSIC \& ESPRIT are largely used algorithms for DOA estimation.

\section{RELATED WORK}

DOA estimation uses the information got by array elements to discover a DOA of arrival signal. Array arrangement can be a receiving antenna structure that has ability to alter beam patterns by using internal feedback controls during its operation. Directions of incoming arrival signal \& interferers are got using a DOA algorithm. DOA calculations can be then utilized by array sensor elements to outline the beam former in a unique approachto maximize radiated power towards signal of interest\& to stifle interference. Thus angular resolution subspaced techniques like MUSIC\& Modified MUSIC algorithms most broadly used

\section{PROPOSED ALGORITHM}

\subsection{Design Considerations}

- Data formulation for ESPRIT and MUSIC.

- Covariance matrix.

- Eigen value and Eigen matrix.

- Signal \& noise subspace. 


\subsection{Description of the Proposed ESPRIT Algorithm:}

The acronym of ESPRIT is Estimation of Signal Parameters via Rotational Invariance Techniques is subspaced DOA calculation method. This algorithm used to discover DOA of incoming signal of various sources. The block diagram for executing ESPRIT as shown below.

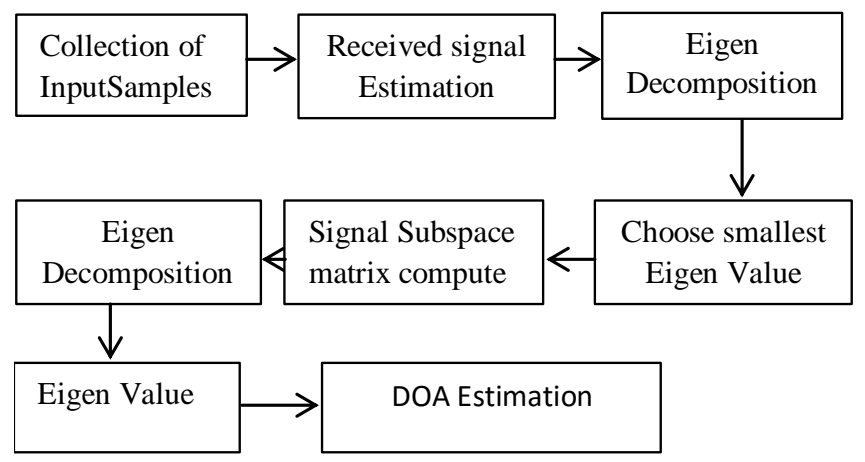

Fig 1. ESPRIT block diagram

\subsection{Mathematical model of ESPRIT}

The steps to perform ESPRIT given as below

Step 1 Collecting a samples \& covariance

$$
R_{x x}=\frac{1}{k} \sum_{k=0}^{K-1} X_{k} \cdot X_{k}^{H}
$$

2: Eigen decomposition

$$
R_{x x} E=E \bigwedge
$$

Where $E$ is diagonal matrices of corresponding vector $\wedge$ is the eigenvalues

3: Smallest Eigen value given by a taking a minimum calculation of the Step 2

4: Subspaced matrix given as below

$$
\text { vector }=\left[\begin{array}{ll}
\text { vector } 1 & \text { vector } 2 \\
\text { vector } 3 & \text { vector } 4
\end{array}\right]
$$

5: DOA can be given as

$$
D O A=\cos ^{-1}\left[\frac{\arg (\text { digonal elements })}{\beta d}\right]
$$

Where $\beta$ is distance between each sensor $\mathrm{d}$ is total distance

\subsection{Description of Proposed MUSIC Algorithm}

MUSIC is acronym which means Multiple Signal classification. This is determination numerical space DOA strategy that provides calculations of fluctuate of signal arriving, accordingly to course of entry. The algorithmic principle depends absolutely on expelling Eigen form of info fluctuation grid.

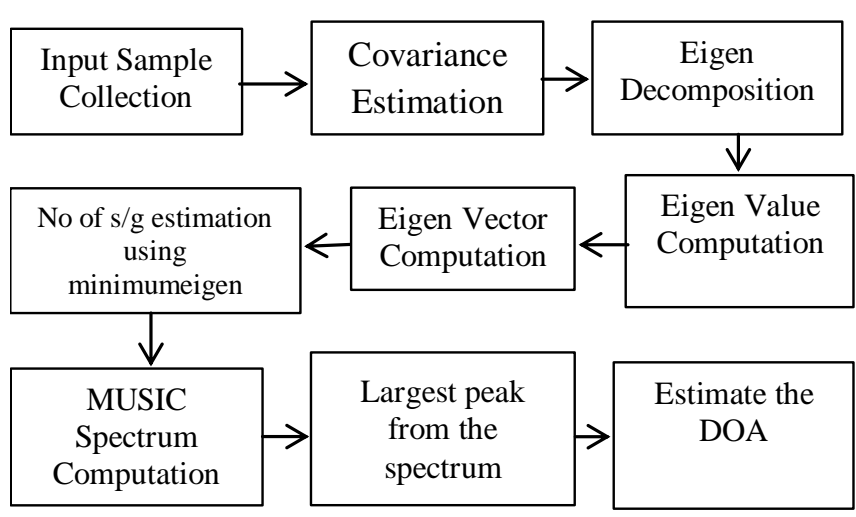

Fig 2. MUSIC algorithm block diagram

Above calculation utilized to illustrate Experimental and theoretical procedure's involved in defining parameters of various signal coming at antenna arrays arrangement from measurement made on a signal obtained at array elements. The steps for executing MUSIC algorithm will be summarized as below:

\subsection{Mathematical Model of MUSIC}

Step 1:samples taken as $X_{k}$ estimate covariance matrix

$$
\hat{R}_{X X}=\frac{1}{k} \sum_{K=0}^{K=-1} X_{K} \cdot X_{K}^{H}
$$

2:Execute Eigen decomposition on $\hat{R}_{x x}$

$$
\hat{R}_{x x} E=E \Lambda
$$

Where $\Lambda=$ diagonal elements of Eigen matrixE $=$ diag $\left(Q_{0}, Q_{1}, \ldots Q_{M-1}\right)$ are the corresponding Eigen vector of $\hat{R}_{x x}$

3: Calculate No. of signals $\widehat{L}$ from a multiplicity $\mathrm{K}$, of a smallest eigenvalue $\lambda_{\min }$ as an equation $\hat{L}=M-K$

4:Calculatespectrum of MUSIC by following Equation.

$$
\widehat{P}_{\text {MUSIC }}(\theta)=\frac{A^{H}(\theta) A(\theta)}{A^{H}(\theta) E_{n} E_{n}^{H} A(\theta)}
$$

Step 4: Obtain the highest peaks of $\hat{P}_{\text {MUSIC }}(\theta)$ to get DOA of arriving incoming signal.

\section{SIMULATION RESULTS}

We analysed performance of a DOA computation with several source positions \& various noise levels where a ESPRIT \& MUSIC algorithm are implemented. In all analysis, it is assumes the same no. of sensors used unless mentioned otherwise. 
A simulation carried by using anmatlab to evaluate ESPRIT \& MUSIC algorithms. In the simulation, we assume that three uncorrelated narrow band signals impinges on a linear array antenna at angles of arrivals $-45,0$ and 45 . The three signals are assumed to have power of $1 \mathrm{~W}, 1.5 \mathrm{~W}$ and $2 \mathrm{~W}$, the no. of sources considered is $\mathrm{N}=12$. Detected signals computed using two algorithms ESPRIT \&MUSIC against different angles are shown in following figures for various conditions. It is obvious fact that the detected highest peaks indicating the DOA of the three incoming signals typically agree with the incident signals.

\subsection{ESPRIT Simulation Results}

DOA of Signals: The graph shown below represents arrival of source incoming signals with noise, In the graph high peaks clearly represents the arrival of various incoming signals at an array elements\& other lower peaks represents noise in the surrounding environment.

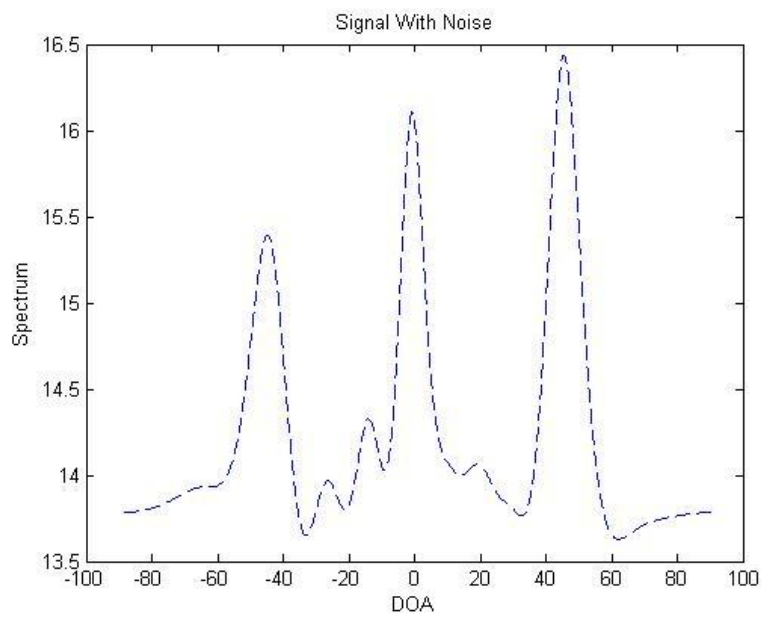

Fig. 3. DOA of Signals with noise of ESPRIT

Spatial spectrum: The graph shown below is used for representing a noise in surrounding environment. As distance between array sensors increases gradually noise will also increases.

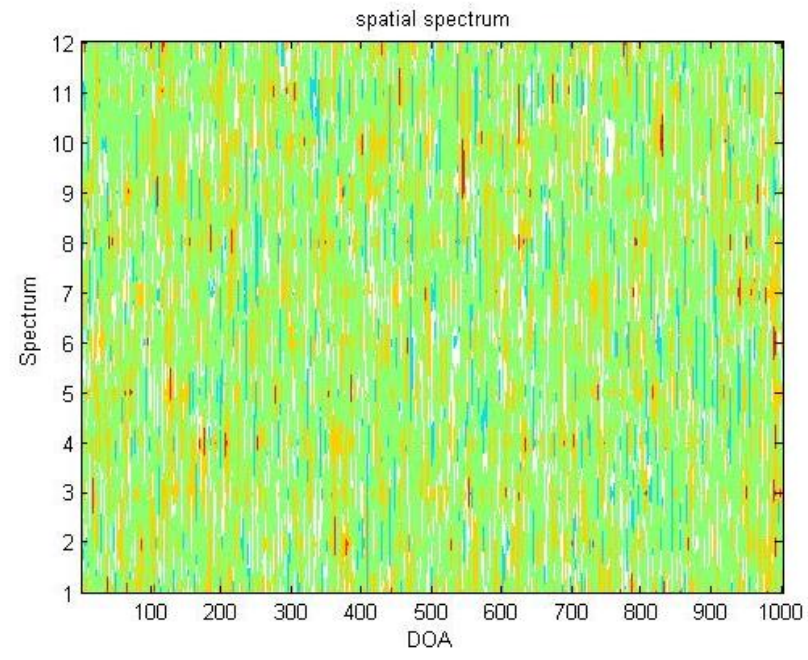

Fig. 4. ESPRIT spatial spectrum
Spatial covariance: The below shown figure represents thespatial covariance matrice of ESPRIT. In case of spatial covariance as no. of array sensors increases accordingly noise surrounding an incoming arriving signals also increase gradually as shown in below graph.

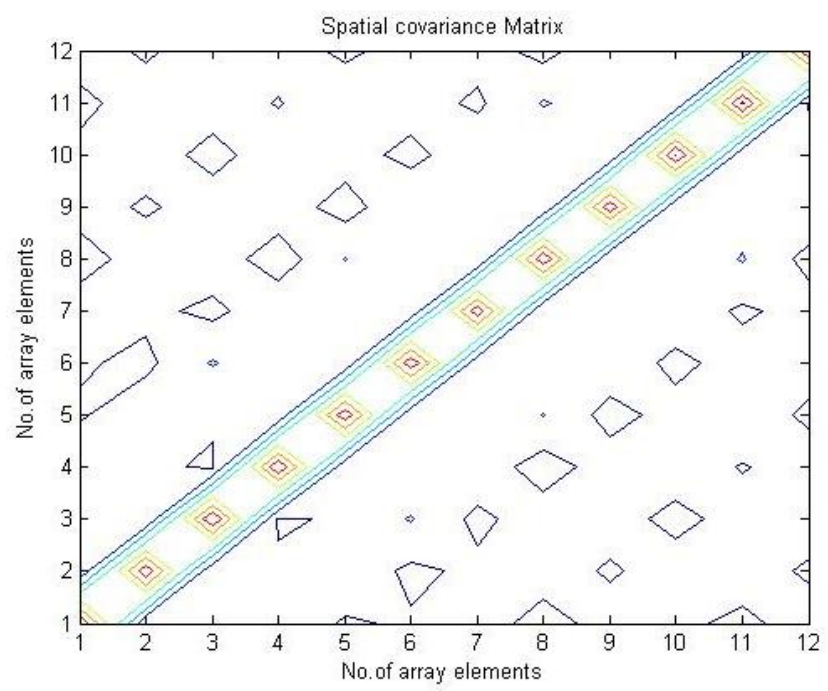

Figure. 5. Spatial covariance Matrix

Coherent Spectrum: The below graph shown below represents coherent spectrum of incoming arriving signal.In coherent spectrum pseudo spectrum increases gradually the angle in degree also increase accordingly, hence the accuracy also increases.

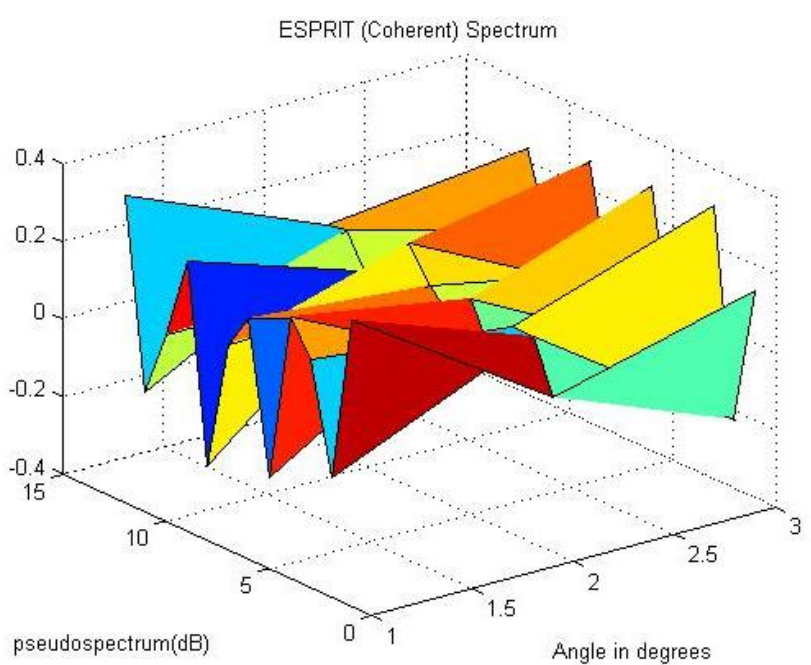

Fig 6. Coherent Spectrum

DOA Estimation: The below shown graph clearly represents Final DOA computation using ESPRIT algorithm. The peaks shown in this graph clearly represents arrival of the incoming signals at array elements. 


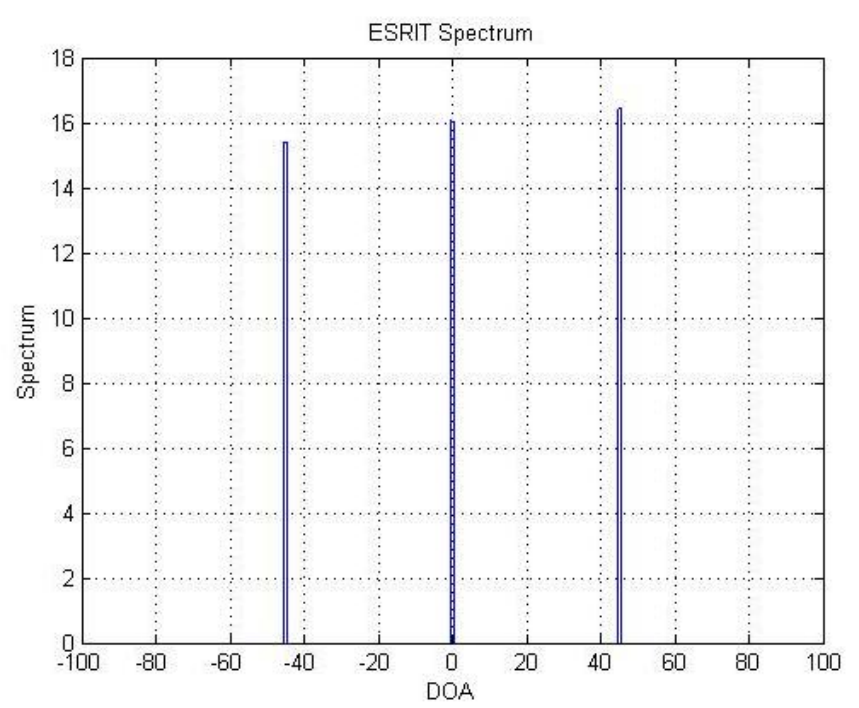

Fig. 7. DOA computation using ESPRIT spectrum

\subsection{MUSIC Simulation Results}

DOA of signals: In MUSIC, the graph shown below precisely represent arrival of arriving signal in occurence of white noise, high peaks shown in graph clearly describes the arrival of incoming signal at array elements and remaining other lower peaks in graph represents noise in the environment.

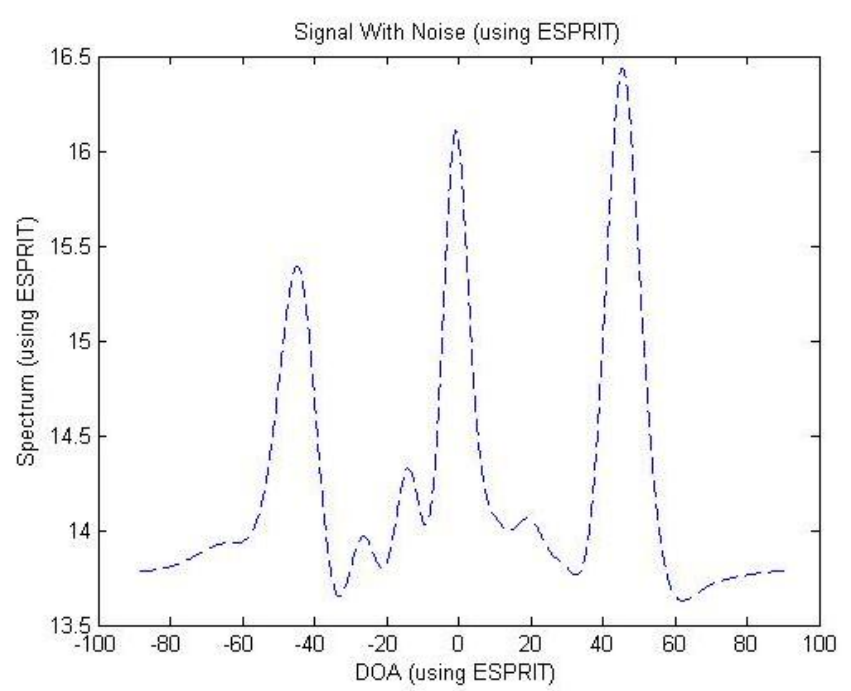

Fig. 8. DOA of Signals with noise of MUSIC

Spatial spectrum: The spectrum shown below clearly used to represent the noise present in surrounding environment. As distance between array elements increases gradually noise also increases accordingly.

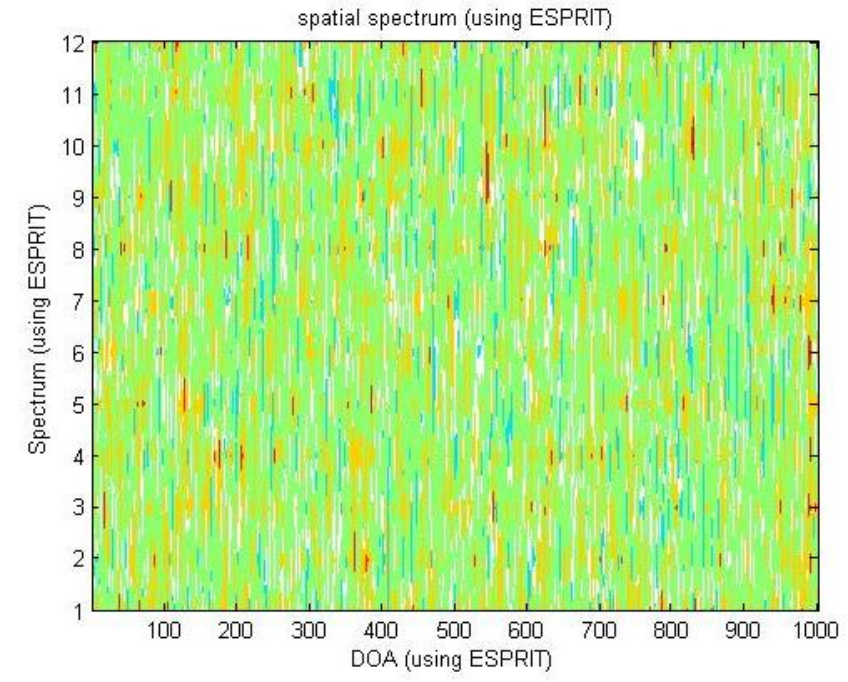

Fig. 9. MUSIC spatial spectrum

Spatial covariance: The below shown figure represents spatial covariance in MUSIC algorithm. In case of spatial covariance as the number of array elements of sytem increases noise also gradually increases gradually as shown below

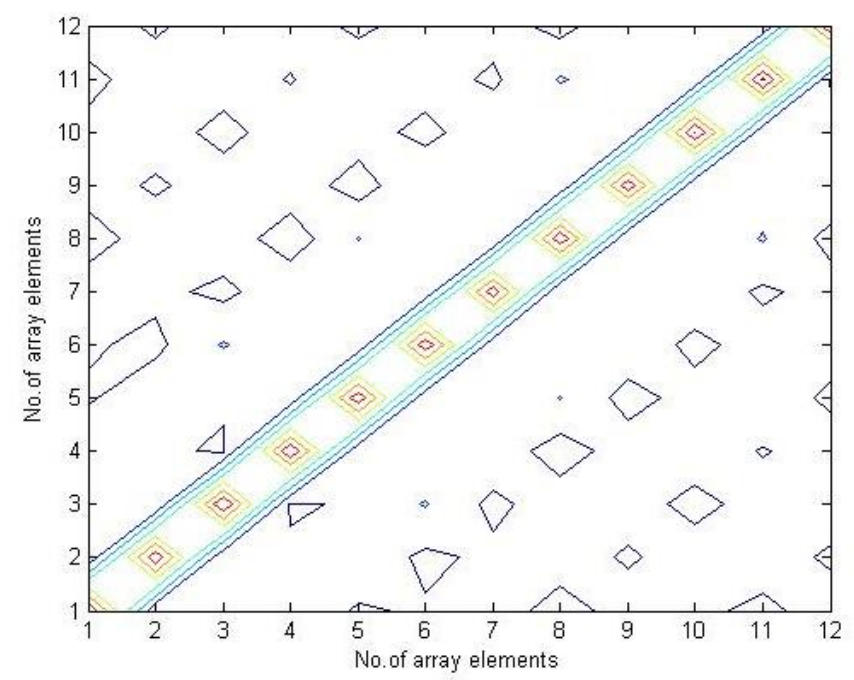

Fig. 10. Spatial covariance of Matrix

Coherent spectrum: In figure shown below clearly represents MUSIC coherent spectrum. In coherent spectrum as pseudo spectrum increases gradually along the axes the angle in degree also increase along, as describe in below fig hence the accuracy of incoming signal increases. 


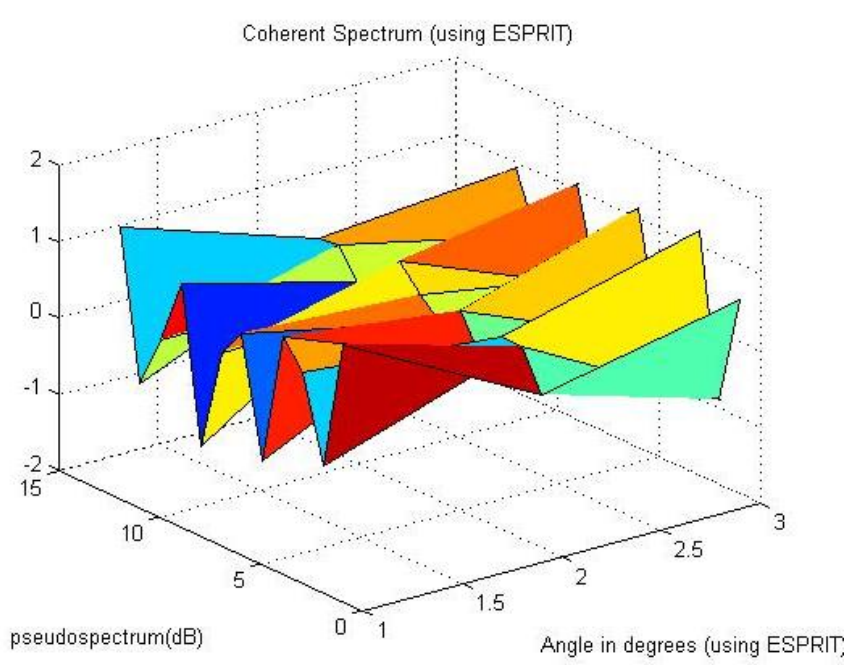

Fig 11. Coherent Spectrum

DOA Estimation: The graph shown below clearly represents the Final DOA computation of MUSIC algorithm. The peaks seen in this graph clearly, represents arrival of incoming signals at array elements.

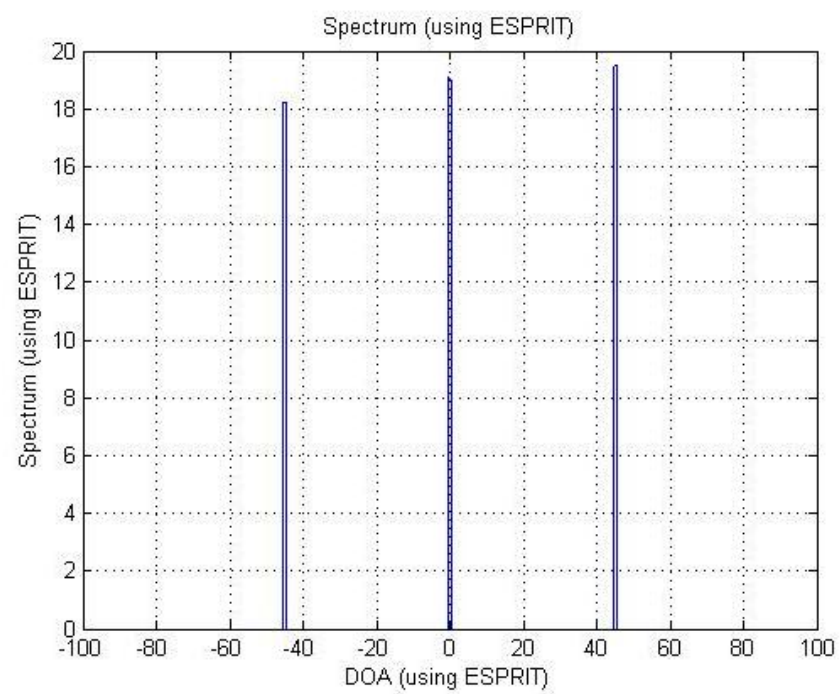

Fig. 12. DOA Estimation of MUSIC spectrum

\section{CONCLUSION}

The ESPRIT and MUSIC performed usingEigen values and Eigen vectors of the incoming signal and noises to calculateDOA of the arriving signal. It becomes easier to isolate the signals from noise as the Eigen vectors for signal $\&$ noise subspace that are orthogonal to each other. It works very efficiently when signals that are being captured on the array of sensors are non-coherent. Efficiency of ESPRIT and MUSIC estimation techniques can enhanced by increasing array element spacing, increasing the no. of array sensors, no. of snapshots and improving the incidence angle difference between the incoming signals.

\section{REFERENCES}

[1]. Ioannides, P; Ballanis, C.A.; -Uniform Circular Arrays for Smart Antennas, ॥, Antennas and Propagation Magazine, IEEE, vol.47, no.4, pp. 192-206, Aug 2005.

[2].Gabriel, W., "Special issue on Adaptive Antennas," Antennas and Propagation, IEEE Transactions on , vol.24, no.5, pp. 573- 574, Sep 1976

[3].Ohira,T."Analog smart antennas: an overview," Personal, Indoor and Mobile Radio Communications, 2002. The 13th IEEE International Symposium on , vol.4, no., pp. 1502- 1506 vol.4, 15-18 Sept. 2002.

[4].Butler J., and Lowe R., -Beam-Forming Matrix Simplifies Design of Electronically Scanned Antennas, Electronic Design, pp. 170-173, April 12, 1961.

[5].Kobayashi, O., Ohira, T., and Ogawa, H., -A novel butler matrix based beam forming network architecture for multiple antenna beam steering\|, IEICE Trans. Electronics, E82-C, 7, pp. 1195-1201, July 1999.

[6].]Gaubatz, D.A., "FFT-Based Analog Beamforming Processor," 1976 UltrasonicsSymposium , vol., no., pp. 676681, 1976.

[7]. M.S. Bartlett, "Periodogram analysis and continuous spectra," Bio-metrica, vol. 37, no. 1/2, pp. 1-16, Jun. 1950.

[8].Capon, J. , "High-resolution frequency-wavenumber spectrum analysis," Proceedings of the IEEE, vol.57, no.8, pp. 1408-1418, Aug. 1969.

[9].Schmidt, R., "Multiple emitter location and signal parameter estimation," Antennas and Propagation, IEEE Transactions on , vol.34, no.3, pp. 276- 280, Mar 1986.

[10].Roy, R., and Kailath, T., "ESPRIT-estimation of signal parameters via rotational invariance techniques," Acoustics, Speech and Signal Processing, IEEE Transactions on , vol.37, no.7, pp.984-995, Jul 1989

[11].Michael Panique, - Design and evaluation of test bed software for a smart antenna system supporting wireless communication in rural areas", Master's Thesis, Montana State University, Dept. of Electrical and Computer Engineering, 2008.

\section{BIOGRAPHIES}

${ }^{1}$ Mr. Prashil M. Junghare has completed his postgraduation degree (M.Tech) in communication engineering from VIT University in 2009. He currently pursuing Ph.D. Degree from PRIST University, Tamilnadu. His area of interest in underwater sensor and navigation.

${ }^{2}$ Mr. Nagaraj, Student, Dept. of ECE, pursuing M.TechIn VLSI Design \& Embedded Systems, The Oxford College of Engineering, Bangalore, India. His area of interest in the field of Under water Communication, Signal Processing, VLSI. He has done his internship in Karnataka Power Corporation Limited (KPCL) and has attended various Conferences. 
${ }^{3}$ Dr. Cyril Prasanna Raj P, Currently working as a Dean (R\&D) at M.S Engineering College, Bangalore. He has completed his Ph.D. degree from Coventry University, UK in 2011. His area of interest in VLSI, Image \& signal processing.

${ }^{4}$ Mrs. Chandrakala S, Currently is working as Assistant professor, Dept. of ECE, The Oxford College of Engineering, Bangalore, India. She has completed hisM.Tech degree from PDA college engineering in 1991, Gulbarga, India. Her area of interest in Power Electronics, VLSI. 